Lymphology 53 (2020) 63-75

\title{
REVIEW OF THE FUNCTION OF SEMA3A IN LYMPHATIC VESSEL MATURATION AND ITS POTENTIAL AS A CANDIDATE GENE FOR LYMIPHEDEMA: ANALYSIS OF THREE FAMIILIES WITH RARE CAUSATIVE VARIANTS
}

M. Ricci, C. Daolio, B. Amato, S. Kenanoglu, D. Veselenyiova, D. Kurti, A. Dautaj, M. Baglivo, S.H. Basha, S. Priya, R. Serrani, M. Dundar, J. Krajcovic, M. Bertelli

Division of Rehabilitation Medicine (MR,RS), Azienda Ospedaliero-Universitaria, Ospedali Riuniti di Ancona, Italy; Pediatric Unit (CD), Carlo Poma Hospital, Mantova, Italy; Department of Clinical Medicine and Surgery (BA), University Federico II of Naples Naples, Italy; Department of Medical Genetics (SK,MD), Faculty of Medicine, Erciyes University, Kayseri, Turkey; Department of Biology (DV,JK), Faculty of Natural Sciences, University of Ss. Cyril and Methodius ivn Trnava, Trnava, Slovakia; MAGI Euregio (SK,DV,DK,MB,MB), Bolzano, BZ, Italy; MAGI-Balkan (DK,AD), Tirana, Albania; EBTNA-Lab (AD,MB), Rovereto, TN, Italy; Innovative Informatica Technologies (SHB,SP), Hyderabad, India; MAGI's Lab (MB), Rovereto, TN, Italy

\section{ABSTRACT}

SEMA3A is a semaphorin involved in cell signaling with PlexinA1 and Neuropilin-1 (NRP1) receptors and it is responsible for recruiting dendritic cells into lymphatics. Mutations in the SEMA3A gene result in abnormalities in lymphatic vessel development and maturation. We investigated the association of SEMA3A variants detected in lymphedema patients with lymphatic maturation and lymphatic system malfunction. First, we used NGS technology to sequence the SEMA3A gene in 235 lymphedema patients who carry wild type alleles for known lymphedema genes. We detected three different missense variants in three families. Bioinformatic results showed that some protein interactions could be altered by these variants. Other unaffected family members of the probands also reported different episodes of subclinical edema. We then evaluated the importance of the SEMA3A gene in the formation and maturation of lymphatic vessels. Our results determined that SEMA3A variants segregate in families with lymphatic system malformations and recommend the inclusion of SEMA3A in the gene panel for testing of patients with lymphedema.

Keywords: SEMA3A, semaphorins, lymphedema, NGS, genetic diagnosis

SEMA3A (semaphorin 3A, also known as collapsin-1) is a member of the large SEMA family of proteins that have important roles in the development of many physiological systems (1). The gene encoding SEMA3A maps to chromosome $7(7 q 21.11)$ and is a large gene composed of 25 exons. Like other semaphorins, SEMA3A has a role in nervous system development, although the SEMA3A gene is also expressed in organs such as the lungs, kidneys, and lymphatic vessels.

Semaphorins were first identified for their effect on axon growth and orientation in the 
early 1990s (2). There are eight major classes of semaphorins, identified by their sequence similarities and structures $(3,4)$. The first two semaphorin classes are defined in invertebrates, classes 3-7 in vertebrates and class 8 in viruses (4). SEMA3A is a class 3 semaphorin. All semaphorins have a long SEMA domain with about 500 amino acid residues. Structural analysis of the SEMA domain has shown its critical function in dimerization and regulating receptor binding $(5,6)$.

SEMA3A interacts with PlexinA1 and Neuropilin-1 (NRP1) receptors to recruit dendritic cells into lymphatics (7). It is the first recognized ligand of Neuropilin receptors and has a role in various biological processes $(8,9)$. Neuropilins are transmembrane proteins of about 900 amino acids and are the most studied plexin co-receptors. SEMA3A is not capable of initiating plexin signaling by itself, hence it binds to NRP1 or NRP2 receptors to trigger the signal (10). In the absence of SEMA3A, plexin is deactivated and no signaling occurs. Binding of SEMA3A activates plexin signaling; the signal is transmitted through the cell to regulate various biological processes, such as cardiovascular development, hemangiogenesis, and lymphangiogenesis $(11,12)$.

Although different semaphorins are expressed in blood and lymphatic vessels, SEMA3A takes part in maturation of lymphatic vessels. Its function in the lymphatic system is closely linked to its receptor NRP1. High levels of NRP1 are found in the perivascular cells (pericytes) of lymphatic vessels. Pericytes coat lymphatic vessels during vessel and valve development and are responsible for vascular homeostasis and permeability (13).

Several studies have been carried out on animal models (Table 1). The first studies on $\mathrm{Sema}_{3} \mathrm{a}^{-/}$mice showed that they survived for three weeks, then died of sudden heart failure (14). Lymphatic phenotype was not considered in this study.

Bouvrée et al showed increased expression of the Nrp1 receptor in lymphatic vessels. This facilitates tight binding of the Sema3a protein to lymphatic valves. It has been shown that mice carrying a mutation in the binding site of NRP1 (Nrp1 ${ }^{\text {sema3a-/- }}$ ) do not bind Sema3a and have small valves that are abnormally coated in smooth muscle cells (8). Experiments also demonstrated that obstruction of Sema3a binding to Nrp1 leads to malformation of lymphatic valves (12). Impaired flow of lymphatic fluid has also been reported in Sema3a $^{-/-}$mice (15).

Interstitial fluid is transported from the tissues to the bloodstream by the lymphatic system, which consists of a network of lymphatic vessels with intraluminal valves that prevent backflow (8). Lymphatic vessel and valve malformations can cause lymphedema, a progressive condition arising from abnormal accumulation of interstitial fluid. Symptoms of lymphedema include swelling of the extremities, associated with susceptibility to fibrosis and infections (16). Since lymphedema has a considerable effect on quality of life, early detection of genetic variants by screening is

TABLE 1

Sema3a Gene - Mouse Models and Associated Phenotypes

\begin{tabular}{|l|l|l|l|l|}
\hline Organis & Gene & Function & Lethality & Lymphatic phenotypes \\
\hline Mouse & Sema3a & $\begin{array}{l}\text { Protein } \\
\text { coding }\end{array}$ & $\begin{array}{l}\text { Sema3a }{ }^{-/} \text {mice } \\
\text { survived to 3 weeks, } \\
\text { then died of sudden } \\
\text { heart failure }\end{array}$ & $\begin{array}{l}\text { 14. } \\
\text { coated in SMCs }^{8} \text {, impaired flow of lymphatic } \\
\text { fluid }^{15} \text {. }\end{array}$ \\
\hline
\end{tabular}


aimed at reducing the devastating effects of the disease and at guiding treatment (17).

To our knowledge, SEMA3A variants have not yet been associated with lymphedema, but rather with neurological and inflammatory diseases. Villa et al associated two SEMA3A SNPs (rs36026860 and rs28469467) with predisposition for Alzheimer's disease (18).

Liu et al showed that two variants (rs12707682 and rs7804122) may be associated with clinical manifestations of systemic lupus erythematosus (19), an autoimmune disease characterized by inflammation of different organs. SEMA3A is believed to be implicated in predisposition for systemic lupus erythematosus because it inhibits $T$ cell proliferation and therefore plays a critical role in the immune system (19).

Here we performed NGS analysis of DNA from Italian lymphedema patients to investigate whether $S E M A 3 A$ has a role in lymphatic system malfunction and the development of lymphedema.

\section{MATERIALS AND METHODS}

\section{Clinical Evaluation}

Caucasian patients $(n=246)$ diagnosed with lymphedema in different hospitals across Italy were included retrospectively in the study. No consanguinity was reported in their families. Clinical diagnosis of lymphedema was according to accepted criteria and confirmed by three-phase lymphoscintigraphy according to the protocol of Bourgeois. Genetic testing was performed on germline DNA extracted from saliva or blood of the proband. Segregation analysis was performed on DNA extracted from saliva of probands' relatives. Participants are aware by approved specific informed consent that more genes will be investigated in addition to the standard diagnostic procedure. It is also declared that the integrative results of diagnostic genetic test are to be considered currently without approved clinical value. The study was conducted in accordance with the principles of the Declaration of Helsinki and it was approved by the local Ethics Committee.

\section{Genetic Analysis}

A custom-made oligonucleotide probe library was designed to capture all coding exons and flanking exon/intron boundaries ( $\pm 15 \mathrm{bp}$ ) of 29 genes known to be associated with lymphedema. We added the candidate gene SEMA3A to our panel.

Genetic variants with likely clinical significance (pathogenic, likely pathogenic and of unknown significance according to ACMG guidelines), identified in probands, were confirmed by bidirectional Sanger sequencing on a CEQ8800 Sequencer (Beckman Coulter). Segregation of heterozygous variants in SEMA3A in available family members was performed by Sanger sequencing.

We developed a Next-GenerationSequencing (NGS) protocol for screening the most frequent genes known to be associated with lymphedema, namely ADAMTS3 (OMIM 605011), CELSR1 (OMIM 604523), EPHB4 (OMIM 600011), FAT4 (OMIM 612411), FLT4 (OMIM 136352), FOXC2 (OMIM 602402), GATA2 (OMIM 137295), GJA1 (OMIM 121014), GJC2 (OMIM 608803), HGF (OMIM 142409), KIF11 (OMIM 148760), PIEZO1 (OMIM 611184), PTPN14 (OMIM 603155), SOX18 (OMIM 601618), and VEGFC (OMIM 601528), including the candidate gene SEMA3A (OMIM 603961).

We searched the international databases, dbSNP (www.ncbi.nlm.nih.gov/SNP/) and Human Gene Mutation Database professional (HGMD; http://www.biobase-international. com/product/hgmd), for all nucleotide changes. In-silico evaluation of the pathogenicity of nucleotide changes in exons was performed using the Variant Effect Predictor tool (http://www.ensembl. org/Tools/ VEP) and MutationTaster (http://www. mutationtaster.org). Minor allele frequencies (MAF) were checked in the Genome 
Aggregation Database (gnomAD) (http:// gnomad.broadinstitute. org/). All variants were evaluated according to American College of Medical Genetics and Genomics guidelines (20). Detailed pre-test genetic counseling was provided to all subjects, who were then invited to sign informed consent to use their anonymized genetic results for research.

\section{In Silico Analysis}

The primary amino acid sequence of SEMA3A in FASTA format (Table 2) was used as target sequence to search the Swiss model template library (SMTL) version 2019-10-24 and Protein Data Bank (PDB) release 2019-10-18 (21) with BLAST (Basic Local Alignment Search Tool) (22) and HHBlits (23) for matching evolution-related structures. Models were built based on target-template alignment using ProMod3 of the SWISSMODEL server (24). Coordinates conserved between target and template were copied from the template to the model. Insertions and deletions were remodeled using a fragment library. Side chains were then rebuilt. Finally, the geometry of the model was regularized by the CHARMM27 force field (25). If loop modelling with ProMod3 failed, an alternative model was built with PROMOD-II (26). Global and per-residue model quality was assessed using the QMEAN scoring function (27). BioVia Discovery Studio Visualizer ver17.2 (28) was used to visualize the modeled protein, to vary the targeted amino acids and to analyze interactions at molecular level.

\section{RESULTS}

\section{Clinical and Genetic Evaluation}

We collected DNA samples of 246 lymphedema patients to investigate whether

TABLE 2

Clinical Features of Probands and Family Members with Rare SEMA3A Variants

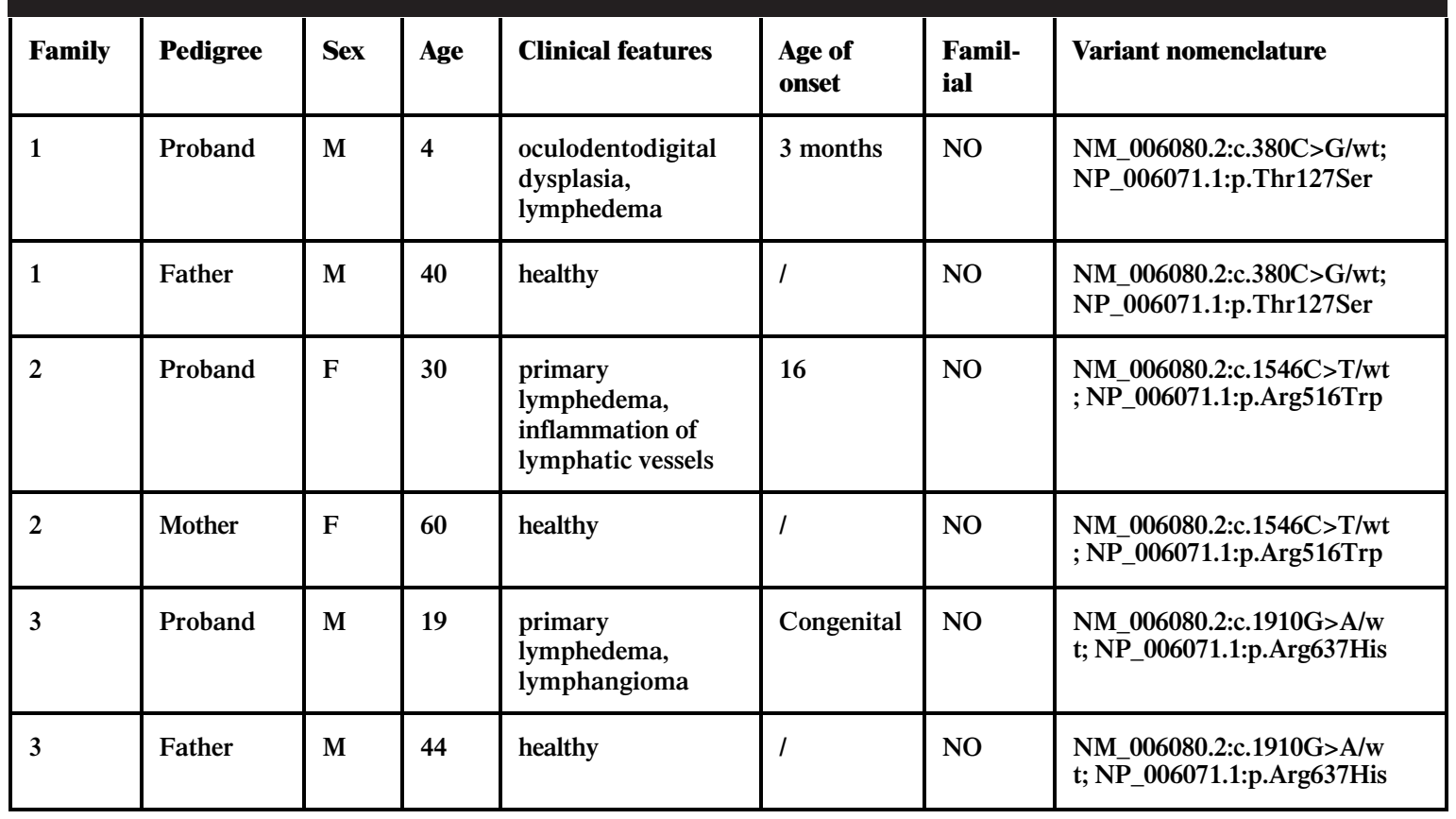




\begin{tabular}{|l|l|l|l|l|}
\hline \multicolumn{5}{|c|}{ Summary of SEMA3A Variants in our Cohort } \\
\hline Variant & dbSNP ID & SIFT & PolyPhen & Frequency \\
\hline $\begin{array}{l}\text { SEMA3A:NM_006080.2:c.38 } \\
\text { 0C>G: } \\
\text { NP_006071.1:p.Thr127Ser }\end{array}$ & Not available & $\begin{array}{l}\text { deleterious } \\
(0.02)\end{array}$ & $\begin{array}{l}\text { possibly damaging } \\
(0.99)\end{array}$ & Not available \\
\hline $\begin{array}{l}\text { SEMA3A:NM_006080.2:c.15 } \\
\text { 46C>T: }\end{array}$ & rs148900275 & $\begin{array}{l}\text { deleterious } \\
\text { NP_006071.1:p.Arg516Trp }\end{array}$ & $\begin{array}{l}\text { probably damaging } \\
(0.997)\end{array}$ & $\begin{array}{l}\text { 0.00009386 } \\
\text { Total } \\
\text { frequency } \\
0.00009234\end{array}$ \\
\hline $\begin{array}{l}\text { SEMA3A:NM_006080.2:c.19 } \\
\text { 10G>A: } \\
\text { NP_006071.1:p.Arg637His }\end{array}$ & rs766918295 & $\begin{array}{l}\text { deleterious } \\
(002)\end{array}$ & $\begin{array}{l}\text { probably damaging } \\
(0.991)\end{array}$ & $\begin{array}{l}0.00002847 \\
\text { Total } \\
\text { frequency } \\
0.00003539\end{array}$ \\
\hline
\end{tabular}

they carry variants in known lymphedema genes. This cohort is currently the second largest in Europe. However, 235 probands tested negative for the variants investigated. We therefore decided to perform a more comprehensive testing of potential lymphedema genes, which included SEMA3A (OMIM 603961), described in this paper. We found rare heterozygous, missense variants in SEMA3A in three probands. All cases were sporadic, since the family members of all probands are healthy. Clinical features of probands and family members with SEMA3A variants are listed in Table 3.

In the first family, we tested the proband and three other family members. The proband, male, 4 years, has oculodentodigital dysplasia and lymphedema, diagnosed at 3 months and carries a rare heterozygous single-nucleotide variant NM_006080.2:c.380C >G. The variant causes an amino acid change from threonine to serine in position 127 of the protein. SIFT and PolyPhen predict this variant to be deleterious (0.02) and possibly damaging (0.99), respectively. The variant is not listed in $\mathrm{dbSNP}$ and the frequency is unknown. The father of the proband carries the same variant (Fig. 1) and his medical history reported mild episodes of edema of the feet. The mother and brother carry wild type alleles of this gene. No other family members have lymphedema.

In the second family, the proband, female, 30 years, was diagnosed with primary lymphedema and inflammation of lymphatic vessels at age 16, and carries a rare heterozygous missense NM_006080.2:c.1546C > T variant (dbSNP rs148900275) which at protein level changes arginine516 to tryptophane. SIFT and PolyPhen predict the variant to be deleterious (0) and probably damaging (0.997), respectively. The variant has a frequency of 0.00009386 according to GnomAD. The mother carries the same variant as the proband, unlike the father and brother. She reported episodes of cyclic edema in the lower limbs.

In the third family, the proband, male, 19 years, was diagnosed at birth with primary lymphedema and lymphangioma and was found to carry a rare heterozygous single nucleotide variant NM_006080.2:c.1910G >A (dbSNP rs766918295) which causes a change from arginine to histidine in position 637 of the protein. The variant is predicted to be deleterious (002) by SIFT, and probably damaging (0.991) by PolyPhen. According to 


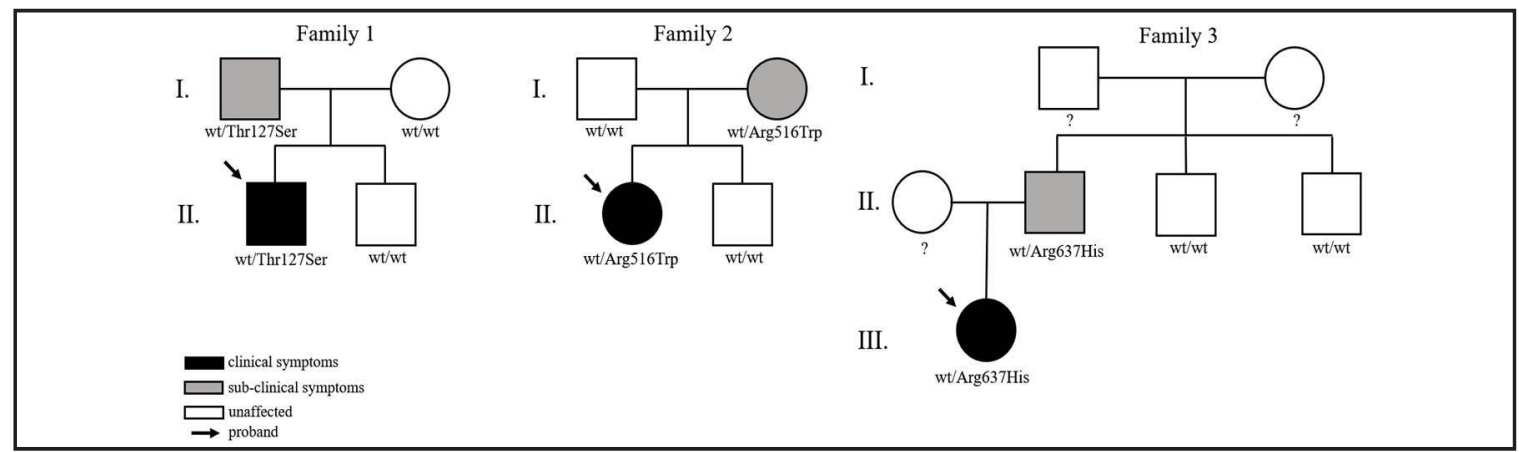

Fig. 1. Pedigrees of families carrying SEMA3A variants.

GnomAD it has a frequency of $\mathbf{0 . 0 0 0 0 2 8 4 7 . ~ T w o ~}$ uncles of the proband (brothers of the proband's father) were tested for the variant and found to be negative, although the father has the same variant as the proband. Whereas father is generally healthy, he reported edema of the lower limbs when subjected to physical exercise. The SEMA3A variants detected in our patients are summarized in Table 4.

In Silico Results, Template Selection and Model Building

Template search with BLAST and HHBlits against the SWISS-MODEL template library (SMTL, last update: 2019-10-24, last included
PDB release: 2019-10-18) produced a total of 10,566 templates that matched the SEMA3A target sequence with different sequence identities and quality percentages. Details of the top 10 templates are shown in Table 5.

Based on the percentage of sequence identity, similarity and best quality square, the 1q47.1.A chain was selected to align the template and query sequences for model building. The model is shown in Fig. 2. The model was then entered in the Discovery studio visualizer to generate the Thr127Ser, Arg516Trp and Arg637His variants.

Molecular level interaction analysis between interacting native/variant residues produced the snapshots shown in Figs. 3-5. Details of

TABLE 4

Primary Amino Acid Sequence Used to Search for Templates in Order to Build Models for the SEMA3A Protein

MGWLTRIVCLFWGVLLTARANYQNGKNNVPRLKLSYKEMLESNNVITFNGLANSSSYHTF LLDEERSRLYVGAKDHIFSFDLVNIKDFQKIVWPVSYTRRECKWAGKDILKECANFIKVLK AYNQTHLYACGTGAFHPICTYIEIGHHPEDNIFKLENSHFENGRGKSPYDPKLLTASLLIDG ELYSGTAADFMGRDFAIFRTLGHHHPIRTEQHDSRWLNDPKFISAHLISESDNPEDDKVYFF FRENAIDGEHSGKATHARIGQICKNDFGGHRSLVNKWTTFLKARLICSVPGPNGIDTHFDE LQDVFLMNFKDPKNPVVYGVFTTSSNIFKGSAVCMYSMSDVRRVFLGPYAHRDGPNYQWV PYQGRVPYPRPGTCPSKTFGGFDSTKDLPDDVITFARSHPAMYNPVFPMNNRPIVIKTDVNY QFTQIVVDRVDAEDGQYDVMFIGTDVGTVLKVVSIPKETWYDLEEVLLEEMTVFREPTAISA MELSTKQQQLYIGSTAGVAQLPLHRCDIYGKACAECCLARDPYCAWDGSACSRYFPTAKRR TRRQDIRNGDPLTHCSDLHHDNHHGHSPEERIIYGVENSSTFLECSPKSQRALVYWQFQRRN EERKEEIRVDDHIIRTDQGLLLRSLQQKDSGNYLCHAVEHGFIQTLLKVTLEVIDTEHLEELL HKDDDGDGSKTKEMSNSMTPSQKVWYRDFMQLINHPNLNTMDEFCEQVWKRDRKQRRQ RPGH TPGNSNKWKHLQENKKGRNRRTHEFERAPRSV 


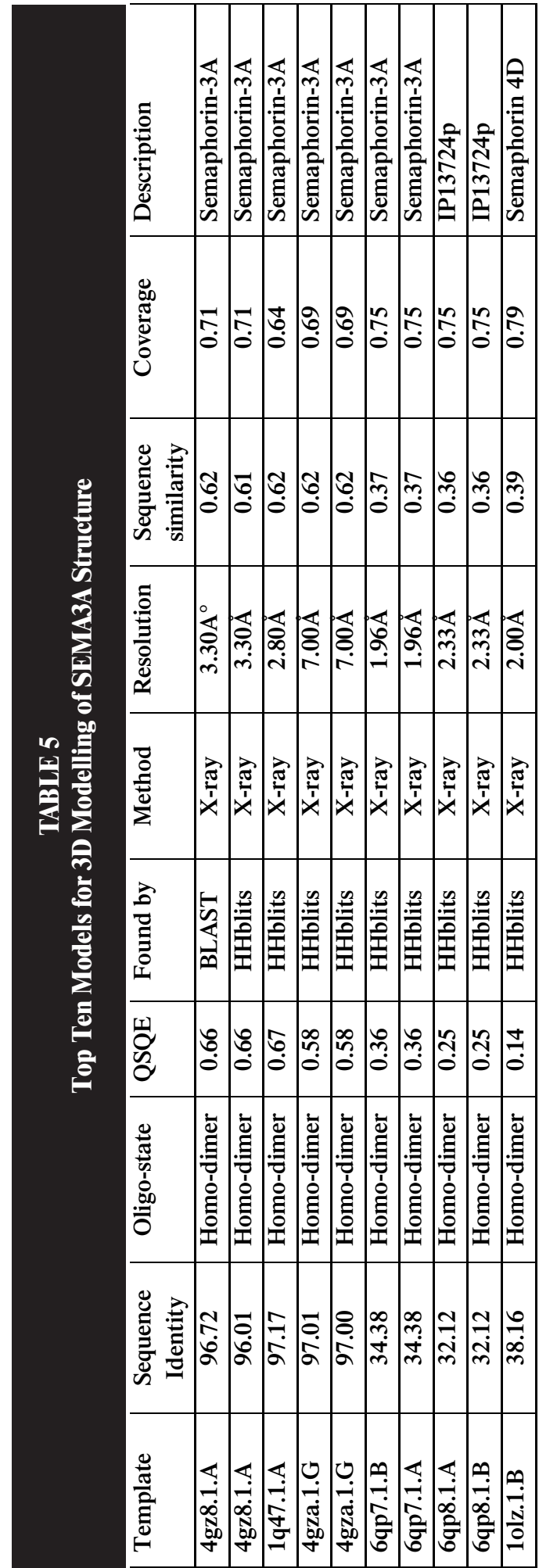

interacting residues, the types of bond they formed and bond lengths in Angstrom units are shown in Tables 6, 7 and 8, respectively.

\section{DISCUSSION AND CONCLUSIONS}

The lymphatic vascular system has essential functions in tissue fluid homeostasis and immune surveillance (29). Abnormalities in lymphatic vessels and other components of the lymphatic system can cause lymphedema and other inflammatory and autoimmune diseases (30-33).

The development of the lymphatic vasculature is well conserved in mammals and has been studied in mouse models. In mice, lymphatic vessels form from endothelial cells in mid-gestation. In the following sequence, endothelial cells migrate and form the primitive lymph sacs by E14.5. Primary lymphatic vessels sprout from the lymphatic sacs and mature into functional lymphatic vessels (34). The Sema3a gene has important functions in the development and maturation of lymphatic vessels.

The findings of several mouse studies suggest a relationship between lymphatic system maturation and Sema3a. In a mouse model, Jurisic et al (12) demonstrated that Sema3a, which can function as a chemorepulsant or chemoattractant, is expressed by lymphatic vessels. They also reported that semaphorin receptor Neuropilin-1 (Nrp1) is expressed in perivascular cells lining the lymphatic vessels during vascular development (12). The authors showed that mice treated in utero with an antibody that prevents interaction of Sema3a and Nrp1 exhibit lymphatic system dysfunction due to morphological abnormalities of lymphatic vessels and valves (12). These results confirm the role of Sema3a/ Nrp1 signaling in normal lymphatic function.

Semaphorins have a crucial role in the movement of cells involved in the immune response. In particular, Sema3a is expressed by dendritic cells, regulating interactions between them and lymphatic endothelial cells. 

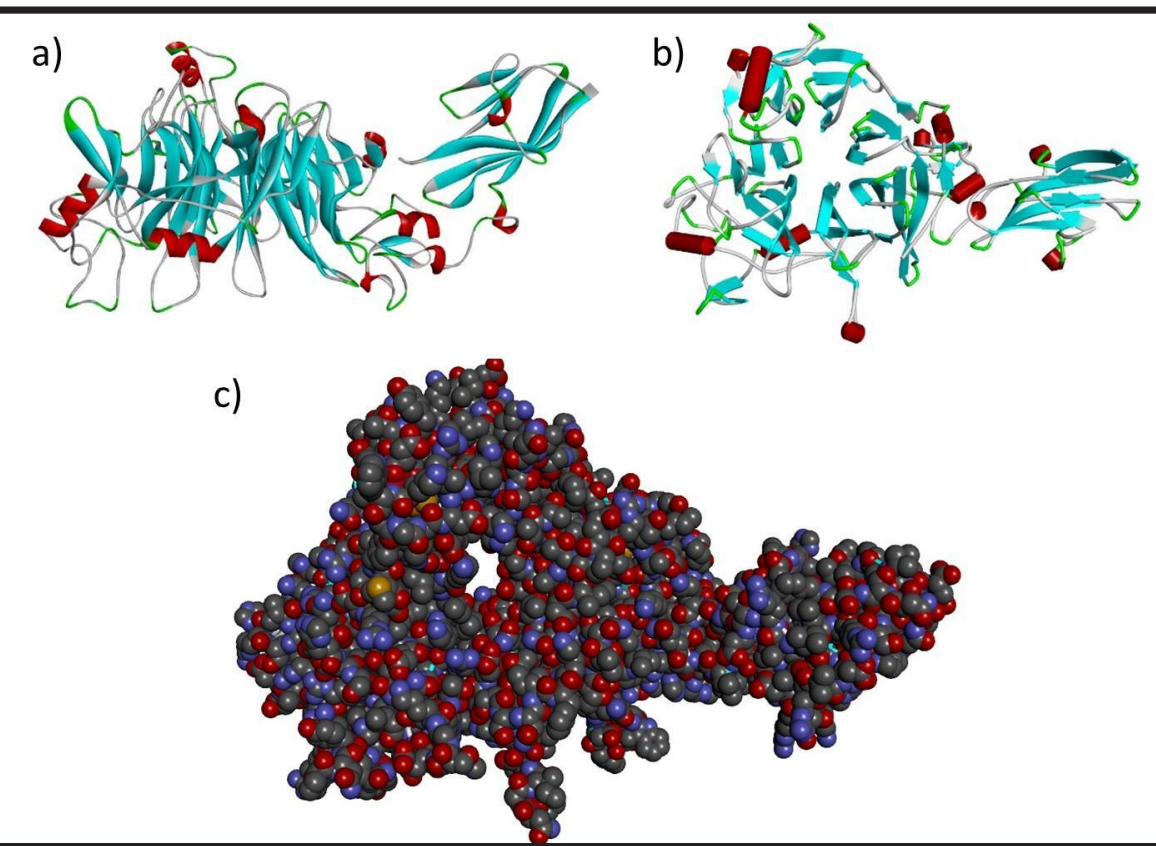

Fig. 2. Modeled structure of SEMA3A gene in a) ribbon b) schematic and c) CPK view. Cyan regions are beta sheets, white represents loops and red represents alpha helices. CPK view: Carbon is shown in grey, oxygen in red, nitrogen in blue, hydrogen in white, chlorine in green, sulfur in yellow, phosphorus in orange, and iron in reddish brown.
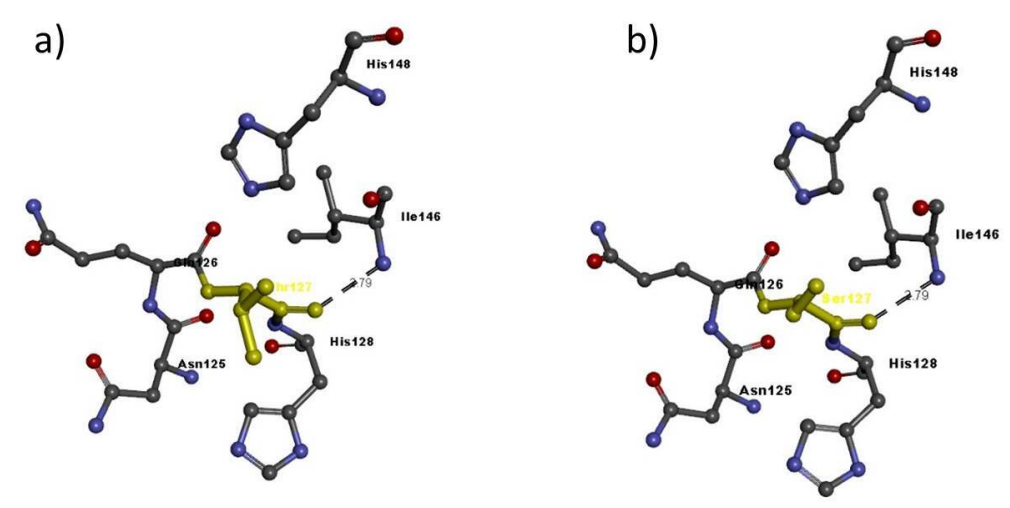

Fig. 3. Molecular interactions of a) Thr127 and b) Ser27 (in yellow) of modeled SEMA3A protein with adjacent residues.

Dendritic cells are antigen-presenting cells important for immune response (35), which under normal conditions are located in peripheral tissues. After encountering antigens, dendritic cells migrate into lymphoid organs where they activate T-cells (36). These findings show that Sema3a is involved in the regulation of cell mobility and morphology (7).
SEMA3A dysfunction has been shown to impair lymph flow, which may lead to build up of this protein-rich fluid in peripheral tissues, a condition known as lymphedema.

Lymphedema is a debilitating disease characterized by regional accumulation of lymph. It affects patients physiologically and psychologically. In genetic testing of 


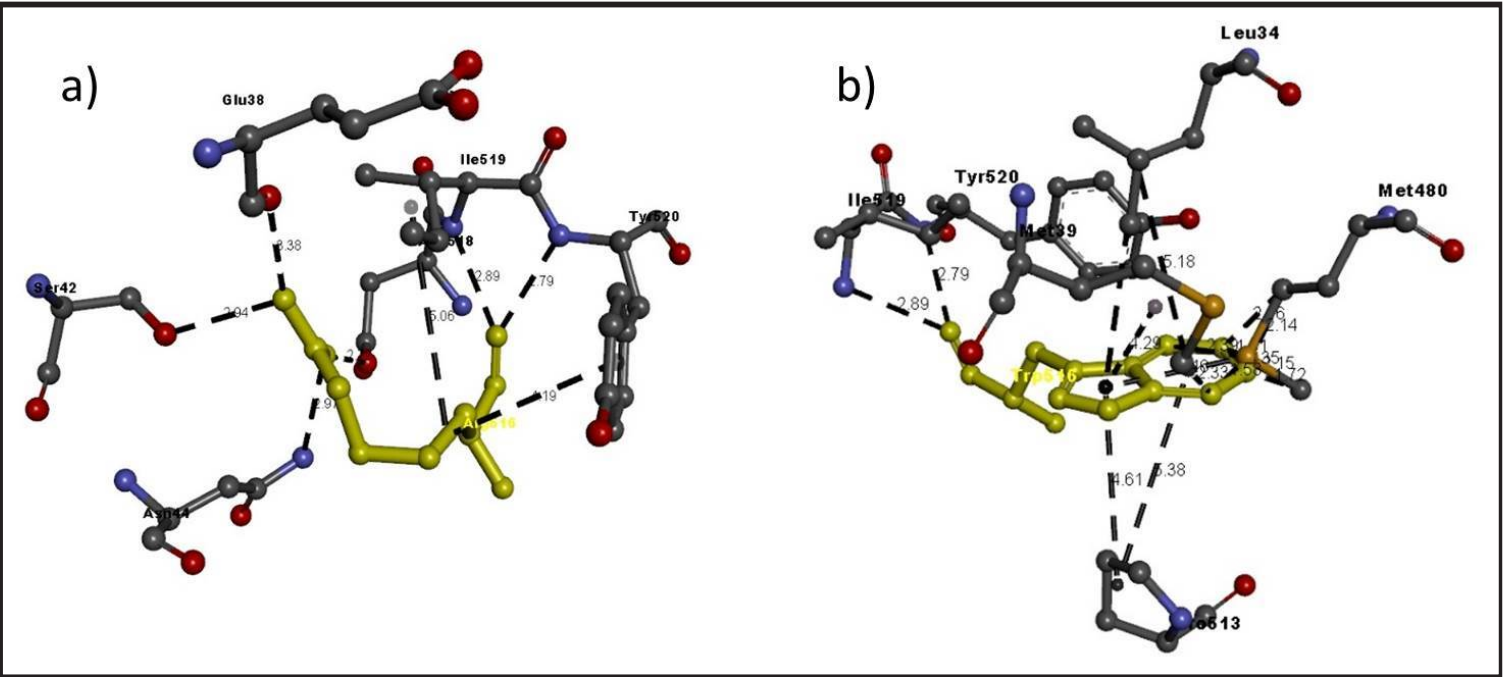

Fig. 4. Molecular interactions of a) Arg516 and b) Trp516 (in yellow) of modeled SEMA3A protein with adjacent residues.
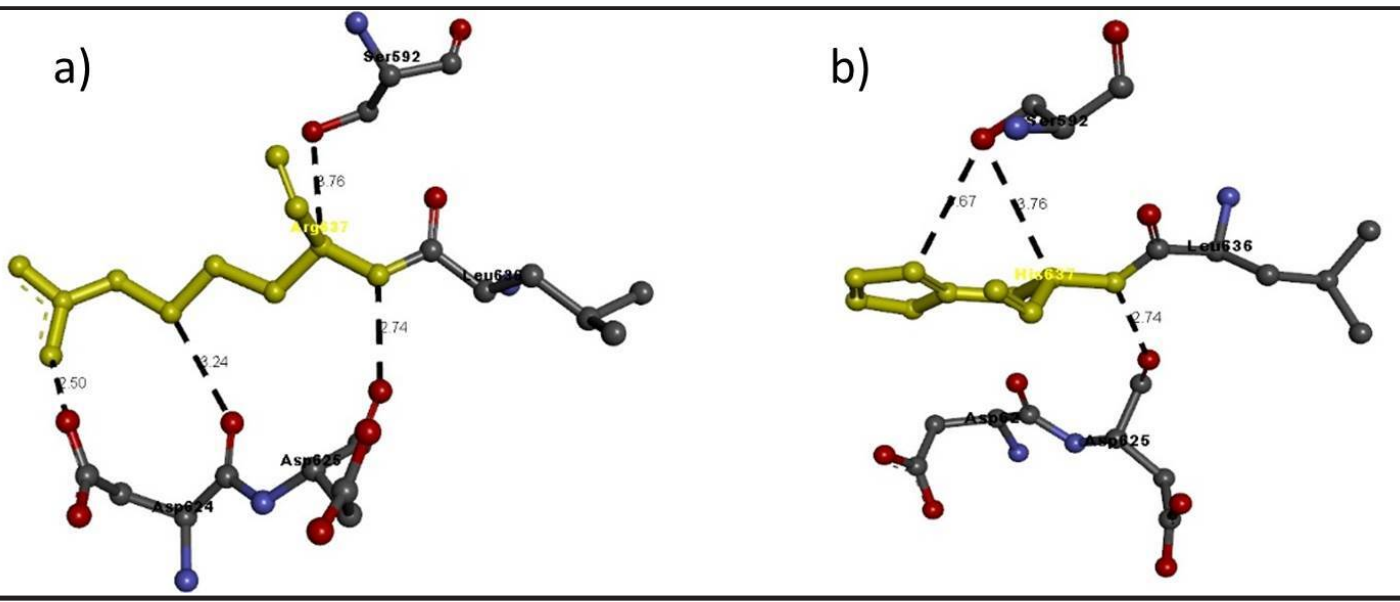

Fig. 5. Molecular interactions of a) Arg637 and b) His637 (in yellow) of modeled SEMA3A protein with adjacent residues.

\begin{tabular}{|c|c|c|c|c|c|}
\hline \multicolumn{6}{|c|}{$\begin{array}{c}\text { TABLE } 6 \\
\text { Details of Molecular Interactions of a) Thri27 and b) Ser127 of Modeled SEMA3A } \\
\text { Protein with Adjacent Residues }\end{array}$} \\
\hline S.No & Mutation & Amino acid & Molecular interactions & $\begin{array}{l}\text { Bond length } \\
\text { in Angstroms }\end{array}$ & Bond type \\
\hline 1. & Thr127Ser & Thr127 & Ile146: N - Thr127: O & 2.79 & H-bond \\
\hline 2. & & Ser127 & Ile146: N - Ser127: O & 2.79 & H-bond \\
\hline
\end{tabular}


TABLE 7

Details of Molecular Interactions of a) Arg516 and b) Trp516 of Modeled SEMA3A Protein with Adjacent Residues

\begin{tabular}{|c|c|c|c|c|c|}
\hline S.No & Mutation & $\begin{array}{l}\text { Amino } \\
\text { acid }\end{array}$ & Molecular interactions & $\begin{array}{l}\text { Bond } \\
\text { length in } \\
\text { Angstroms }\end{array}$ & Bond type \\
\hline 1. & \multirow{25}{*}{ Arg516Trp } & \multirow{8}{*}{ Arg516 } & Arg516: N - Asp518: O & 2.72 & H-bond \\
\hline 2. & & & Arg516: N - Glu38: O & 3.38 & H-bond \\
\hline 3. & & & Arg516: N - Ser42: O & 2.94 & H-bond \\
\hline 4. & & & Ile519: N - Arg516:O & 2.89 & H-bond \\
\hline 5. & & & Tyr520: N - Arg516: O & 2.79 & H-bond \\
\hline 6. & & & Asn44:N - Arg516:N & 2.97 & H-bond \\
\hline 7. & & & Arg516 - Ile519 & 5.06 & Hydrophobic interaction \\
\hline 8. & & & Tyr520 - Arg516 & 4.19 & Hydrophobic interaction \\
\hline 9. & & \multirow{17}{*}{$\operatorname{Trp516}$} & Met39:C - Trp516:C & 2.33 & H-bond \\
\hline 10. & & & Met480:C - Trp516:C & 2.46 & H-bond \\
\hline 11. & & & Met480:C - Trp516:C & 2.14 & H-bond \\
\hline 12. & & & Met480:S - Trp516:C & $2 . .39$ & H-bond \\
\hline 13. & & & Met480:S - Trp516:C & 1.58 & H-bond \\
\hline 14. & & & Met480:S - Trp516:C & 1.31 & H-bond \\
\hline 15. & & & Met480:S - Trp516:C & 0.35 & H-bond \\
\hline 16. & & & Met480:C - Trp516:C & 2.15 & H-bond \\
\hline 17. & & & Met480:C - Trp516:C & 1.72 & H-bond \\
\hline 18. & & & Ile519: N - Trp516: O & 2.89 & H-bond \\
\hline 19. & & & Tyr520: N - Trp516: O & 2.79 & H-bond \\
\hline 20. & & & Met480:S - A:Trp516 & 3.46 & H-bond \\
\hline 21. & & & Trp516 - A:Leu34 & 5.32 & Hydrophobic interaction \\
\hline 22. & & & Trp516 - A:Met39 & 4.29 & Hydrophobic interaction \\
\hline 23. & & & Trp516 - A:Pro513 & 4.16 & Hydrophobic interaction \\
\hline 24. & & & Trp516 - A:Leu34 & 5.18 & Hydrophobic interaction \\
\hline 25. & & & Trp516 - A:Pro513 & 5.38 & Hydrophobic interaction \\
\hline
\end{tabular}




\begin{tabular}{|c|c|c|c|c|c|}
\hline \multicolumn{6}{|c|}{$\begin{array}{c}\text { TABLE } 8 \\
\text { Details of Molecular Interactions of a) Arg637 and b) His637 } \\
\text { of Modeled SEMA3A Protein with Adjacent Residues }\end{array}$} \\
\hline S.No & Mutation & $\begin{array}{c}\text { Amino } \\
\text { acid }\end{array}$ & Molecular interactions & $\begin{array}{l}\text { Bond length } \\
\text { in Angstroms }\end{array}$ & Bond type \\
\hline 1. & \multirow{7}{*}{ Arg637His } & \multirow{4}{*}{ Arg637 } & Arg637: N - Asp624: O & 2.50 & H-bond \\
\hline 2. & & & Arg637: N - Asp625: O & 2.74 & H-bond \\
\hline 3. & & & Arg637:C - Ser592: O & 3.74 & H-bond \\
\hline 4. & & & Arg637: C - Asp624: O & 3.24 & H-bond \\
\hline 5. & & \multirow{3}{*}{ His637 } & His637: N - Asp625: O & 2.74 & H-bond \\
\hline 6. & & & His637: C - Ser592: O & 3.76 & H-bond \\
\hline 7. & & & His637:C - Ser592: O & 3.67 & H-bond \\
\hline
\end{tabular}

lymphedema patients, a panel of 29 genes known to be associated with lymphedema is currently used (16). Since the test does not always identify a genetic variant, new candidate genes need to be discovered. For example, in our study, 235 out of 246 Italian lymphedema patients tested negative for known lymphedema genes. We therefore performed a second test for possible new lymphedema genes, including SEMA3A, reported here.

Three out of $235(3 / 235 ; 1.277 \%)$ lymphedema patients carried a SEMA3A variant. Two probands are male $(66.66 \%)$ and one is female (33.34\%). All cases are sporadic and have missense heterozygous variants. All probands were diagnosed with lymphedema in childhood.

In the first family, the proband and father carry the same missense SEMA3A variant. The father does not currently have lymphedema, but he reported a history of mild edema of the feet in the past. In the second family, the proband and her healthy mother carry the same heterozygous SEMA3A missense variant. Subclinical cyclic edema was detected in the mother. In the last family, the proband carries a rare $S E M A 3 A$ variant, inherited from his father. The father is not currently affected but he reported subclinical edema under physical strain.

In silico analysis showed that SEMA3A codes a protein with Thr127 which has similar stability to the mutant Ser127 in terms of direct hydrogen bonding with nearby Ile146. Both bonds have a bond length of 2.79 Angstroms. In the case of Arg637His, the mutant has three interactions that approximately match the Arg637 interactions, but Arg637 has two more interactions not found in the mutant His637. In the case of Arg516Trp the mutation introduces a major difference: Arg516 shows eight interactions compared to 17 interactions of the mutant. Except for two interactions, i.e. with Tyr520 and Ile519, the other interactions are quite different from those of Arg516. Our findings suggest that these differences somehow alter overall protein structure due to modified interactions with nearby residues, leading to functional defects in the protein.

SEMA3A and its receptors NRP1, NRP2 and PLXNA1 have a key role in the formation of lymphatic valves and lymphatic vessels 
$(8,12)$. Malformations in the lymphatic vasculature can cause lymphedema by impairing lymphatic function and the flow of interstitial fluid. Understanding the role of semaphorins can lead to the development of therapies targeting the semaphorin signaling pathway (37). Interestingly, the protein encoded by $G J A 1$ is linked to oculodentodigital dysplasia, a phenotype identified in one of the reported cases. Both GJA1 and SEMA3A are involved in the lymphatic valve morphogenesis and function $(8,38)$. In the future, it should be investigated whether there may be a genetic interaction between SEMA3A and GJA1.

Although the molecular mechanisms of the development of lymphatic vessels is still not clearly understood, the literature and our results show that SEMA3A and receptor NRP1 play an important role in lymphatic vasculogenesis and can determine predisposition for lymphedema. In conclusion, the variants in the SEMA3A gene described here can be considered for genetic testing in patients with lymphatic system malformations, irrespective of the manifestation of lymphedema.

\section{ACKNOWLEDGMENTS}

We would like to thank Helen Ampt for English language editing. This work was supported by funding from the Provincia Autonoma di Trento within the initiative LP 6/99 (dgp 1045/2017).

\section{CONFLICT OF INTEREST AND DISCLOSURE}

The authors declare no competing financial interests exist.

\section{REFERENCES}

1. Alto, LT, JR Terman: Semaphorins and their signaling mechanisms. Methods Mol. Biol. 1493 (2017), 1-25. doi:10.1007/978-1-4939-6448-2_1

2. Luo, Y, D Raible, JA Raper: Collapsin: A protein in brain that induces the collapse and paralysis of neuronal growth cones. Cell. 75
(1993), 217-227.

doi:10.1016/0092-8674(93)80064-L

3. Goodman, C, A Kolodkin, Y Luo, A Püschel, J

Raper: Unified nomenclature for the

Semaphorins/Collapsins. Cell. 97 (1999), 551-552. doi:10.1016/S0092-8674(00)80766-7

4. Pasterkamp, RJ, AL Kolodkin: Semaphorin junction: Making tracks toward neural connectivity. Curr. Opin. Neurobiol. 13 (2003), 79-89. doi:10.1016/S0959-4388(03)00003-5

5. Gherardi, E, CA Love, RM Esnouf, EM Jones: The sema domain. Curr. Opin. Struct. Biol. 14 (2004), 669-678. doi:10.1016/j.sbi.2004.10.010

6. Siebold C, EY Jones: Structural insights into semaphorins and their receptors. Semin. Cell Dev. Biol. 24 (2013), 139-145. doi:10.1016/ j.semcdb.2012.11.003

7. Takamatsu, H, N Takegahara, Y Nakagawa, et al: Semaphorins guide the entry of dendritic cells into the lymphatics by activating myosin II. Nat. Immunol. 11 (2010), 594-600. doi:10.1038/ni.1885

8. Bouvrée, K, I Brunet, R Del Toro, et al: Semaphorin3A, Neuropilin-1, and PlexinA1 are required for lymphatic valve formation. Circ. Res. 111 (2012), 437-445. doi:10.1161/ CIRCRESAHA.112.269316

9. Kolodkin, AL, DV Levengood, EG Rowe, et al: Neuropilin is a semaphorin III receptor. Cell. 90 (1997), 753-762. doi:10.1016/ S0092-8674(00)80535-8

10. Cariboni, A, K Davidson, $S$ Rakic, et al: Defective gonadotropin-releasing hormone neuron migration in mice lacking SEMA3A signalling through NRP1 and NRP2:

Implications for the aetiology of hypogonadotropic hypogonadism. Hum. Mol. Genet. 20 (2011), 336-344. doi:10.1093/hmg/ ddq468

11. Pascoe, HG, $Y$ Wang, $X$ Zhang: Structural mechanisms of plexin signaling. Prog. Biophys. Mol. Biol. 118 (2015), 161-168. doi:10.1016/ j.pbiomolbio.2015.03.006

12. Jurisic, G, H Maby-El Hajjami, S Karaman, et al. An unexpected role of Semaphorin3ANeuropilin-1 signaling in lymphatic vessel maturation and valve formation. Circ. Res. 111 (2012), 426-436. doi:10.1161/ CIRCRESAHA.112.269399

13. Avolio, E, VV Alvino, MT Ghorbel, $P$ Campagnolo: Perivascular cells and tissue engineering: Current applications and untapped potential. Pharmacol. Ther. 171 (2017), 83-92. doi:10.1016/ j.pharmthera.2016.11.002

14. Behar, O, JA Golden, H Mashimo, FJ Schoen, MC Fishman: Semaphorin III is needed for normal patterning and growth of nerves, bones and heart. Nature 383 (1996), 525-528. doi:10.1038/383525a0 
15. Ochsenbein, AM, S Karaman, G Jurisic, M Detmar: The role of Neuropilin-1/ Semaphorin 3A signaling in lymphatic vessel development and maturation. In: Developmental Aspects of the Lymphatic Vascular System. 214 (2014), 143-152. doi:10.1007/978-3-7091-1646-3_11

16. Michelini, S, S Paolacci, E Manara, et al: Genetic tests in lymphatic vascular malformations and lymphedema. J. Med. Genet. 55 (2018), 222-232. doi:10.1136/ jmedgenet-2017-105064

17. Rockson, SG: Lymphedema. Am. J. Med. 110 (2001), 288-295. doi:10.1016/ S0002-9343(00)00727-0

18. Villa, C, E Venturelli, C Fenoglio, et al: Candidate gene analysis of semaphorins in patients with Alzheimer's disease. Neurol. Sci. 31 (2010), 169-173. doi:10.1007/ s10072-009-0200-1

19. Liu, LN, P Wang, YF Zou, et al: Semaphorin-3A, semaphorin-7A gene single nucleotide polymorphisms, and systemic lupus erythematosus susceptibility. Autoimmunity 52 (2019), 161-167. doi:10.1080/08916934.2019.1642333

20. Richards, S, N Aziz, S Bale, et al: Standards and guidelines for the interpretation of sequence variants: A joint consensus recommendation of the American College of Medical Genetics and Genomics and the Association for Molecular Pathology. Genet. Med. 17 (2015), 405-424. doi:10.1038/ gim.2015.30

21. Berman, HM, J Westbrook, Z Feng, et al: The Protein Data Bank. Nucleic Acids Res. 28 (2000), 235-242. doi:10.1093/nar/28.1.235

22. Camacho, C, G Coulouris, V Avagyan, et al: BLAST +: Architecture and applications. BMC Bioinformatics 10 (2009), 1-9. doi:10.1186/1471-2105-10-421

23. Remmert, M, A Biegert, A Hauser, J Söding: HHblits: Lightning-fast iterative protein sequence searching by HMM-HMM alignment. Nat. Methods 9 (2012), 173-175. doi:10.1038/nmeth.1818

24. Waterhouse, A, M Bertoni, S Bienert, et al: SWISS-MODEL: Homology modelling of protein structures and complexes. Nucleic Acids Res. 46 (2018), W296-W303. doi:10.1093/nar/gky427

25. Mackerell, AD, M Feig, CL Brooks: Extending the treatment of backbone energetics in protein force fields: Limitations of gas-phase quantum mechanics in reproducing protein conformational distributions in molecular dynamics simulation. J. Comput. Chem. 25 (2004), 1400-1415. doi:10.1002/jcc.20065

26. 26. Guex, N, MC Peitsch, T Schwede: Automated comparative protein structure modeling with SWISS-MODEL and SwissPdbViewer: A historical perspective.

Permission granted for single print for individual use.

Reproduction not permitted without permission of Journal LYMPHOLOGY.
Electrophoresis 30 (SUPPL. 1) (2009), 162-173. doi:10.1002/elps.200900140

27. Benkert, P, M Biasini, T Schwede: Toward the estimation of the absolute quality of individual protein structure models. Bioinformatics 27 (2011), 343-350. doi:10.1093/bioinformatics/ btq662

28. Studio, BD: Dassault Systèmes BIOVIA. Discovery Studio Modeling Environment [Internet].; 2016.

29. Tammela T, K Alitalo: Lymphangiogenesis: Molecular mechanisms and future promise. Cell 140 (2010), 460-476. doi:10.1016/ j.cell.2010.01.045

30. Baluk, P, T Tammela, E Ator, et al: Pathogenesis of persistent lymphatic vessel hyperplasia in chronic airway inflammation. $\mathbf{J}$. Clin. Invest. 115 (2005), 247-257. doi:10.1172/ JCI200522037

31. Kunstfeld, R, S Hirakawa, YK Hong, et al: Induction of cutaneous delayed-type hypersensitivity reactions in VEGF-A transgenic mice results in chronic skin inflammation associated with persistent lymphatic hyperplasia. Blood 104 (2004), 1048-1057. doi:10.1182/blood-2003-08-2964

32. Skobe, M, T Hawighorst, DG Jackson, et al: Induction of tumor lymphangiogenesis by VEGF-C promotes breast cancer metastasis. Nat. Med. 7 (2001), 192-198. doi:10.1038/84643

33. Szuba, A, SG Rockson: Lymphedema: Anatomy, physiology and pathogenesis. Vasc. Med. 2 (1997), 321-326. doi:10.1177/1358863X9700200408

34. Schulte-Merker, S, A Sabine, TV Petrova: Lymphatic vascular morphogenesis in development, physiology, and disease. J. Cell. Biol. 193 (2011), 607-618. doi:10.1083/ jcb.201012094

35. Steinman, RM, J Banchereau: Taking dendritic cells into medicine. Nature 449 (2007), 419-426. doi:10.1038/nature06175

36. Randolph, GJ, V Angeli, MA Swartz: Dendritic-cell trafficking to lymph nodes through lymphatic vessels. Nat. Rev. Immunol. 5 (2005), 617-628. [doi:10.1038/nri1670

37. Epstein, JA, H Aghajanian, MK Singh: Semaphorin signaling in cardiovascular development. Cell Metab. 21 (2015), 163-173. doi:10.1016/j.cmet.2014.12.015

38. Kanady, JD, AM Simon: Lymphatic communication: Connexin junction, what's your function? Lymphology 44 (2011), 95-102.

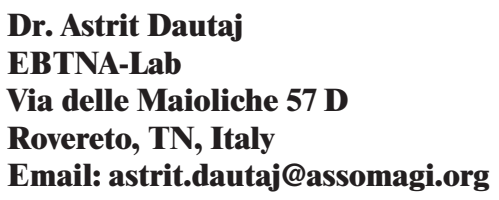

